\title{
Coxsackieviruses B3 infection of myocardial microvascular endothelial cells activates fractalkine via the ERK1/2 signaling pathway
}

\author{
JILI WEN ${ }^{1-3}$ and CONGXIN HUANG ${ }^{1-3}$ \\ ${ }^{1}$ Department of Cardiology, Renmin Hospital of Wuhan University; ${ }^{2}$ Cardiovascular Research Institute, \\ Wuhan University; ${ }^{3}$ Hubei Key Laboratory of Cardiology, Wuhan, Hubei 430060, P.R. China
}

Received May 5, 2017; Accepted September 7, 2017

DOI: $10.3892 / \mathrm{mmr} .2017 .7536$

\begin{abstract}
Infections by pathogens may lead to cardiovascular diseases, including acute/chronic myocarditis. (Coxsackieviruses B3) CVB3 is considered to be the most common causative agent in m-yocarditis, which can lead to dilated cardiomyopathy. The present study aimed to investigate the mechanism of CVB3-infected myocardial microvascular endothelial cells. The CVB3 infection was detected by $50 \%$ tissue culture infective dose $\left(\mathrm{TCID}_{50}\right)$. The role of fractalkine (FKN) in the infection was detected using western blotting and RNA interference. To assess mitogen-activated protein kinase signaling activity, levels of total and phosphorylated extracellular signal-regulated kinase (ERK)1/2, c-Jun N-terminal kinase, and p38 were measured at 0,20,40, and 60 min after CVB3 infection by western blot analysis. The results showed that infection activated FKN via the ERK1/2 signaling pathway. Furthermore, the TCID $_{50}$ of CVB3 in infected cells was lower compared with that in myocardial microvascular endothelial cells following ERK1/2 inhibition and FKN silencing. CVB3 infection of myocardial microvascular endothelial cells activates FKN via the ERK1/2 signaling pathway. These findings represent a foundation for the development of novel methods of treating CVB3-induced myocarditis.
\end{abstract}

\section{Introduction}

Myocarditis is an inflammatory cardiac muscle disease in association with cardiac dysfunction $(1,2)$. The natural history of acute myocarditis varies from complete recovery to severe heart failure, including ventricular arrhythmias and development of dilated cardiomyopathy (DCM) (3). Epidemiological

Correspondence to: Dr Congxin Huang, Department of Cardiology, Renmin Hospital of Wuhan University, Hubei Zhang Road (Formerly Ziyang Road) Wuchang No. 99, Jiefang Road 238, Wuhan, Hubei 430060, P.R. China

E-mail: huangcongxin@vip.163.com

Key words: Coxsackieviruses B3, fractalkine, ERK1/2 studies of viral myocarditis have been limited because the majority of patients have a clinically unapparent course of myocarditis $(4,5)$. However, it is assumed that $2-5 \%$ of patients with viral infection have myocardial involvement $(5,6)$.

In general, myocarditis arises from infectious, toxic, and immunologic causes. Infectious causes of myocarditis include viruses, parasites, protozoa, and fungi, with viruses being the most common cause $(7,8)$. Coxsackieviruses are enteroviruses that can be divided into two classes; class $\mathrm{A}$ is composed of 23 serotypes and class B is comprised of six serotypes. CoxsackievirusB3 (CVB3) is believed to be the most common causative agent of myocarditis, which can lead to DCM $(5,9)$. CVBs are single-stranded RNA viruses that have natural tropisms for epithelial cells, immune cells, and cardiomyocytes (8).

Chemokines are low-molecular-mass cytokines that are classified into the $\mathrm{C}, \mathrm{CC}, \mathrm{CXC}$, and $\mathrm{CX} 3 \mathrm{C}$ families based on the spacing of their N-terminus cysteine residues $(10,11)$. Fractalkine (FKN; CX3CL1), the only member of the CX3C family, is involved in several inflammatory diseases, including cardiovascular diseases (12). FKN exists as membrane-bound and soluble forms; the membrane-bound form is synthesized as a transmembrane molecule with an extracellular N-terminal domain that is attached by a mucin-like stalk to the cell surface, whereas soluble FKN is generated via cleavage at the base of the mucin-like stalk by two metalloproteinases, a disintegrin and metalloproteinase (ADAM) 10 and ADAM17 (12-14).

The mitogen-activated protein kinase (MAPK) signaling pathway links extracellular signals with intracellular targets to control fundamental cellular processes such as proliferation, growth, migration, differentiation, embryogenesis, and death (15). MAPKs consist of three subfamilies, whose members include extracellular signal-regulated kinase 1/2 (ERK1/2), p38, and c-Jun N-terminal kinase (JNK) (16). The significance of MAPK in myocarditis has been highlighted in several reviews. Ribosomal S6 kinases (RSK) comprise a family of serine/threonine kinases that lie at the terminus of the mitogen-regulated ERK-MAPK pathway (17). The stimulation of ERK initiates a cascade of activating events including phosphorylation of RSK by ERK and translocation to the nucleus where they phosphorylate nuclear substrates. Many RSK ( $\mathrm{p}^{90 \mathrm{rsk}}$ contained) substrates have been identified, implicating RSK in a myriad of cellular 
processes $(18,19)$. Previously reported a relationship between myocarditis and the MAPK pathway (20).

Myocardial microvascular endothelial cells was one of the role protected the myocardial, in the procedure of CVB3 infection. How did the myocardial microvascular endothelial cells show its protecting function? And in protected procedure, how the KFN and MAPK expression level changed. In this study, the role of FKN in CVB3 infection of myocardial microvascular endothelial cells were demonstrated, and the KFN and MAPK expression level also were showed.

\section{Materials and methods}

Materials. All chemicals and reagents were purchased from Sigma-Aldrich (Merck KGaA, Darmstadt, Germany), unless otherwise specified. Anti-ERK1/2 (cat. no. 16-284), anti-phospho-ERK1/2 (cat. no. 05-797), anti-JNK (cat. no. 04-210), anti-phospho-JNK (cat. no. 46-613MAG), anti-p38 (cat. no. ABS29), anti-phospho-p38 (Cat. MABS64), anti- $\mathrm{p}^{90 \mathrm{rsk}}$ (cat. no. 04-417) and anti-phospho-p ${ }^{90 \mathrm{rsk}}$ antibodies (cat. no. ABS1849) were purchased from EMD Millipore (Billerica, MA, USA). U0126 (cat. no. CAS 109511-58-2), an ERK1/2 inhibitor, was purchased from Santa Cruz Biotechnology, Inc. (Santa Cruz, CA, USA). CVB3 was provided from China Center for Disease Control and Prevention (Beijing, China).

Myocardial microvascular endothelial cell culture. Human myocardial microvascular endothelial cells (cat. no. 6000) were purchased from ScienCell Research Laboratories (Carlsbad, CA, USA). Cells were cultured at $37^{\circ} \mathrm{C}$ in a humidified atmosphere of $5 \% \mathrm{CO}_{2}$ in air. Cells was grown as a monolayer in RPMI-1640 (Hyclone; GE Healthcare Life Sciences, Logan, UT, USA) containing $10 \%$ fetal bovine serum.

$50 \%$ tissue culture infective dose $\left(T_{C I D_{50}}\right)$ assay. Myocardial microvascular endothelial cells $\left(1 \times 10^{3}\right)$ were seeded into each well of a 96-well plate and incubated at $37^{\circ} \mathrm{C}$ overnight with $5 \% \mathrm{CO}_{2}$, so that the cells were $80 \%$ confluent at the time of infection. CVB3 viral supernatants were collected after $6 \mathrm{~h}$ of cultivation and diluted from $10^{-1}$ to $10^{-7}$. Each dilution was repeated 8 times. After incubation at $37^{\circ} \mathrm{C}$ with $5 \% \mathrm{CO}_{2}$ for $2 \mathrm{~h}$, the diluted supernatant was replaced with $100 \mu 1$ of DMEM supplemented with $2 \%$ FBS. The plates were incubated for 24 to $96 \mathrm{~h}$. The viral titer $\left(\mathrm{TCID}_{50}\right)$ was calculated according to the method of Reed and Muench.

Western blot analysis. Myocardial microvascular endothelial cells were homogenized. Proteins were separated by sodium dodecyl sulfate polyacrylamide gel electrophoresis using $8-12 \% T$-gels. The separated proteins were transferred to a nitrocellulose membrane, which was blocked in $5 \%$ fat-free milk for $60 \mathrm{~min}$ at room temperature. The membrane was incubated with a primary antibody (anti-ERK1/2 1:1,000, anti-phospho-ERK1/2 1:800, anti-JNK 1:1,500, anti-phospho-JNK 1:1,000, anti-p38 1:500, anti-phospho-p38

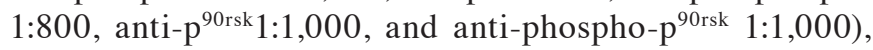
overnight at $4^{\circ} \mathrm{C}$, after which it was washed in PBS and incubated with a species-compatible secondary antibody. Immunoreactive bands were developed using an enhanced chemiluminescent reagent (Pierce; Thermo Fisher Scientific, Inc., Waltham, MA, USA) and quantified by densitometry using Quantity One software (Bio-Rad Laboratories, Inc., Hercules, CA, USA).

RNA interference and cell transfection. To confirm the role of FKN in AngII-induced proliferation of myocardial microvascular endothelial cells, Fkn siRNA was developed to silence Fkn gene expression, as Fkn expression has been found in myocardial microvascular endothelial cells under certain stimuli. Fkn siRNA (sense strand, 5'-GCUGUGGUAGUAAUUCAUAdT dT-3'; antisense strand, 3'-dTdTCGACACCAUCAUUAAGU AU-5') was synthesized by RayBiotech (Guangzhou, China) and transfected into myocardial microvascular endothelial cells using Lipofectamine ${ }^{\circledR} 2000$ (Invitrogen; Thermo Fisher Scientific, Inc.) according to the manufacturer's instructions. siRNA transfection was conducted according to the protocol supplied by Invitrogen. Briefly, $1 \times 10^{5}$ cells were seeded into 6-well plates containing an antibiotic-free medium and incubated overnight. For each well, $5 \mu \mathrm{l}$ siRNA were mixed with $125 \mu$ I OPTI-MEM I. The mixture was then combined with a solution of $5 \mu \mathrm{l}$ lipofectamine in $125 \mu \mathrm{l}$ OPTI-MEM I. After a 20-min incubation period at RT, the mixture was applied to the cells in an appropriate volume of Opti-MEM I so as to achieve a final concentration of $100 \mathrm{nmol} / 1$ for each siRNA. After incubation for $6 \mathrm{~h}$ at $37^{\circ} \mathrm{C}$, RPMI-1640 supplemented with serum was added to the wells. Cells were cultured for an additional $24 \mathrm{~h}$ at $37^{\circ} \mathrm{C}$ before analysis.

Statistical analysis. Differences in protein expression levels between groups were detected using one-way analysis of variance (ANOVA) with SPSS 13.0 and the figure was made by GraphPad Prism 5 software (GraphPad Software, Inc., La Jolla, CA, USA). When the overall F test result of ANOVA was significant, a multiple-comparison Tukey test was applied. Student's t-test was used in two-mean comparisons. Three independent replicates were performed for all experiments. $\mathrm{P}<0.05$ was considered to indicate a statistically significant difference. Data are presented mean \pm standard deviation.

\section{Results}

CVB3 infects myocardial microvascular endothelial cells. The myocardial microvascular endothelial cells which received CVB3 were randomly sacrificed everyday within $96 \mathrm{~h}$ after CVB3 infection as described in the methods section, after which $\mathrm{TCID}_{50}$ values were measured. At $10^{-6.825} / \mathrm{ml}$ of CVB3, approximately $50 \%$ of the myocardial microvascular endothelial cells were not viable; the $\mathrm{TCID}_{50}$ of CVB3 was 6.825 (Fig. 1).

Influence of CVB3 on FKN expression. To assess whether CVB3 treatment altered FKN expression, FKN was detected by western blotting at 0, 20, 40, 60 and 80 min after CVB3 exposure. As shown in Fig. 2, FKN expression was increased at $0,20,40 \mathrm{~min}(\mathrm{P}<0.05)$, but FKN expression was unchanged at 60 and $80 \mathrm{~min}$. FKN protein expression peaked $40 \mathrm{~min}$ after CVB3 infection.

CVB3 activates the MAPK pathway. To assess MAPK signaling activity, levels of total and phosphorylated 


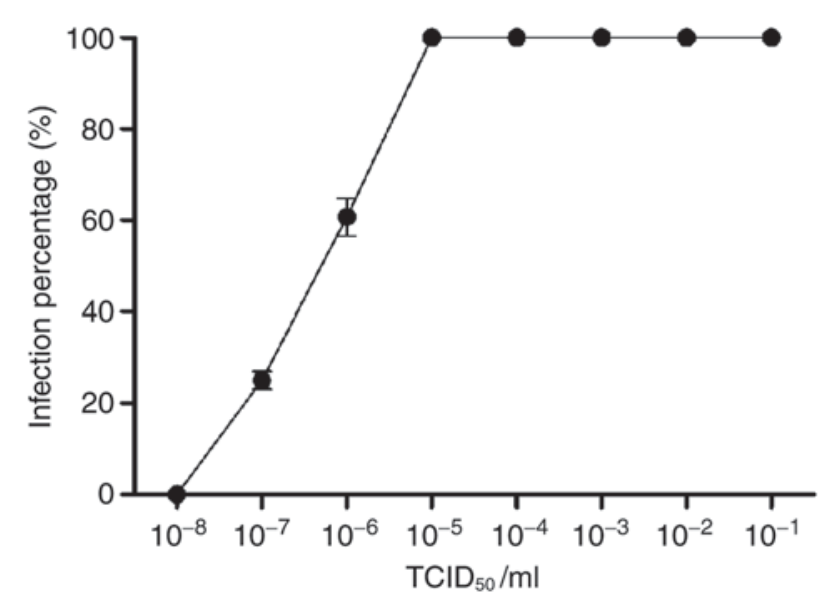

Figure 1. $\mathrm{TCID}_{50}$ of CVB3 in myocardial microvascular endothelial cells. $\mathrm{TCID}_{50}, 50 \%$ tissue culture infective dose; CVB3, Coxsackieviruses B3.
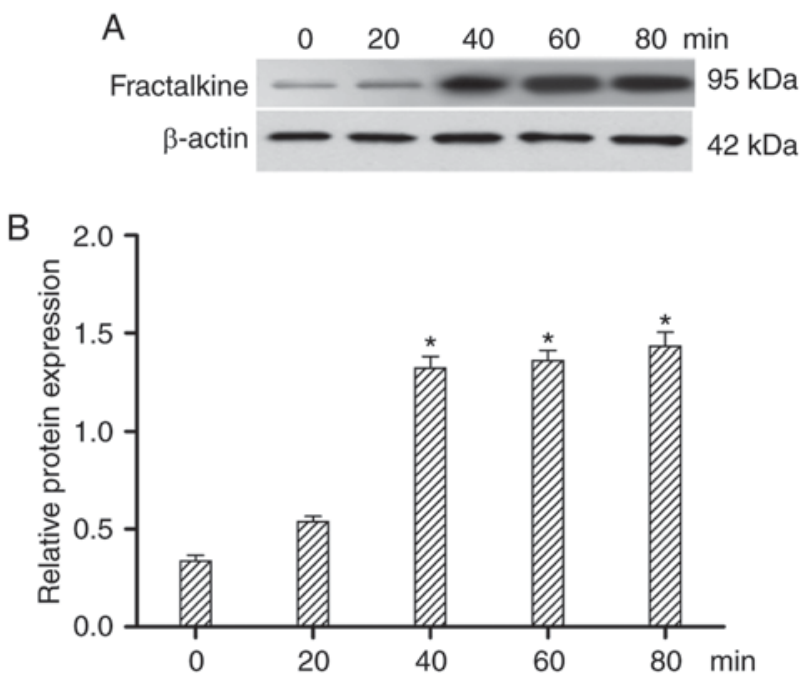

Figure 2. Effects of CVB3 infection on FKN expression. (A) Representative western blots showing levels of FKN. (B) Histograms summarizing the results shown in (A). Results are expressed as mean \pm standard deviation $(\mathrm{n}=5)$. "Significantly different from the 0 min data point $(\mathrm{P}<0.05)$. CVB3, Coxsackieviruses B3; FKN, fractalkine.

ERK1/2, JNK, and p38 were measured 0, 20, 40, 60 and 80 min after CVB3 infection by western blot analysis. Following CVB3 infection, the expression of phosphorylated ERK1/2 was increased at 20, and 40 min, compared with 0 min $(\mathrm{P}<0.05)$, but the expression of phosphorylated ERK1/2 was unchanged between the 60 and 80 min time points (Fig. 3). However, the expression of phosphorylated p-JNK and p-p38 were not changed in all the time. Therefore, ERK1/2 may be the major protein regulating FKN in response to CVB3 infection.

The expression of FKN was reduced following inhibition of ERK1/2 by U0126 (Fig. 4); there was no change in the expression of phosphorylated ERK1/2 and phosphorylated Rsk90 after CVB3 infection (Fig. 5A and B). Moreover, CVB3 showed reduced invasiveness of myocardial microvascular endothelial cells after they were exposed to U0126. At $10^{-7.48} / \mathrm{ml}$ of CVB3, approximately $50 \%$ of the cells were not viable; the $\mathrm{TCID}_{50}$ of CVB3 was 7.48 (Fig. $5 \mathrm{C}$ ).

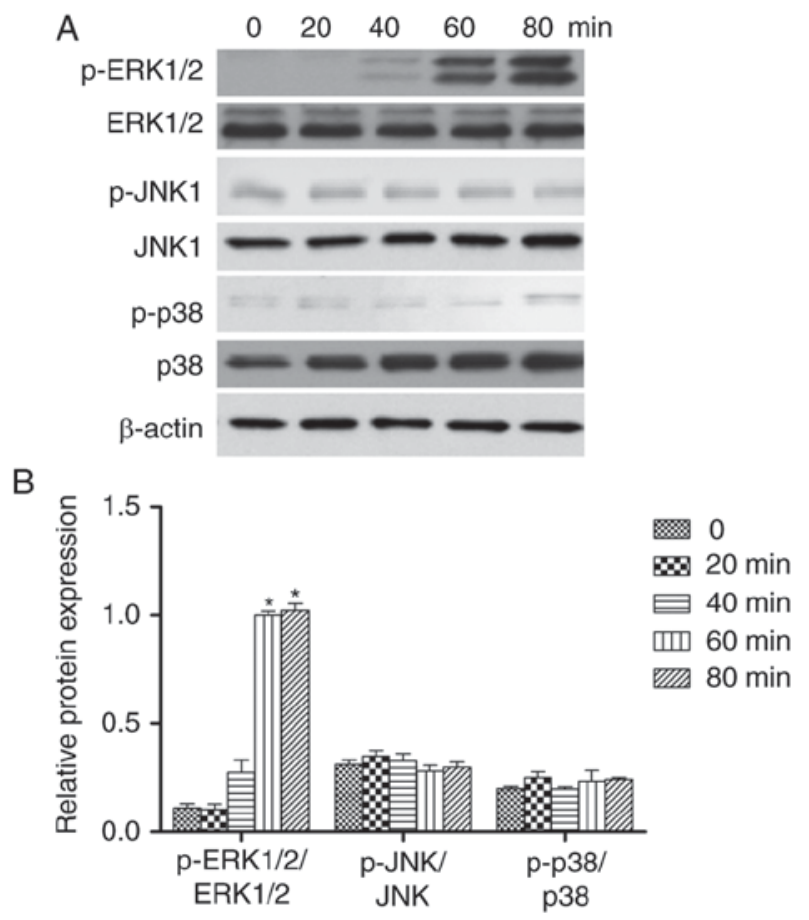

Figure 3. Effect of CVB3 infection on MAPK signaling. (A) Representative western blots showing levels of total and phosphorylated ERK, JNK, and p38. (B) Histograms summarizing the results shown in (A). Results are expressed as mean \pm standard deviation $(\mathrm{n}=5)$. "Significantly different from the 0 min data point $(\mathrm{P}<0.05)$. CVB3, Coxsackieviruses B3; MAPK, mitogen-activated protein kinase; ERK, extracellular signal-regulated kinase; JNK, c-Jun N-terminal kinase.
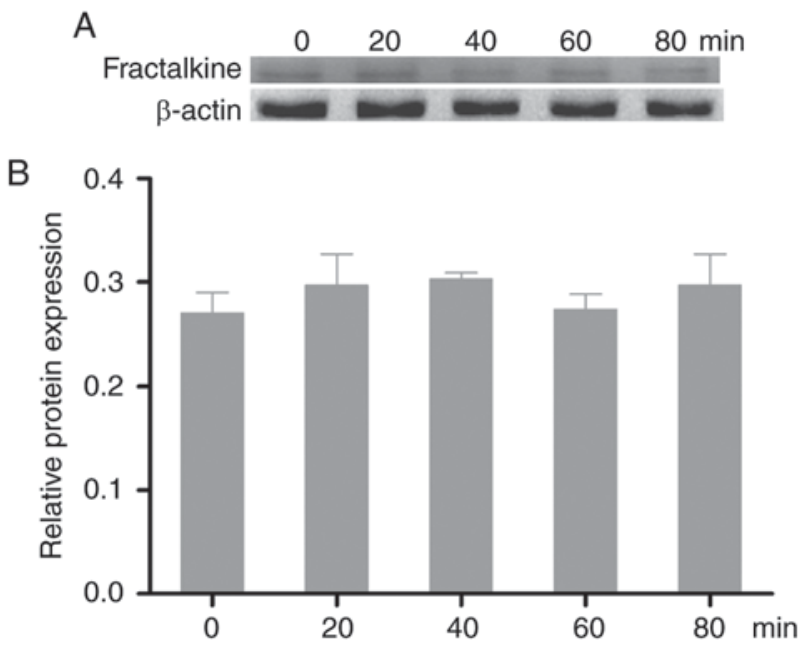

Figure 4. Effect of ERK1/2 inhibition on FKN expression after CVB3 infection. (A) Representative western blots showing levels of FKN. (B) Histograms summarizing the results shown in (A). Results are expressed as mean \pm standard deviation $(\mathrm{n}=5)$. Time 0 means before U0126. CVB3, Coxsackieviruses B3; FKN, fractalkine.

FKN influence the MAPK pathway and CVB3 infectious. To explore the function of FKN in CVB3 infection, FKN translation was inhibited by shRNA. As shown in Fig. 6, there was no difference in the expression of p-ERK1/2, p-JNK, or p-p38 at any time point after FKN was silenced (Fig. 6A and B). FKN shRNA exposure reduced the TCID $_{50}$ of CVB3 to 7.99 (Fig. 6C). 
A

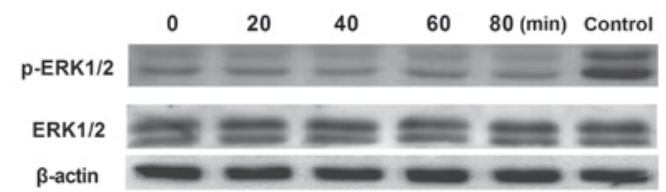

B

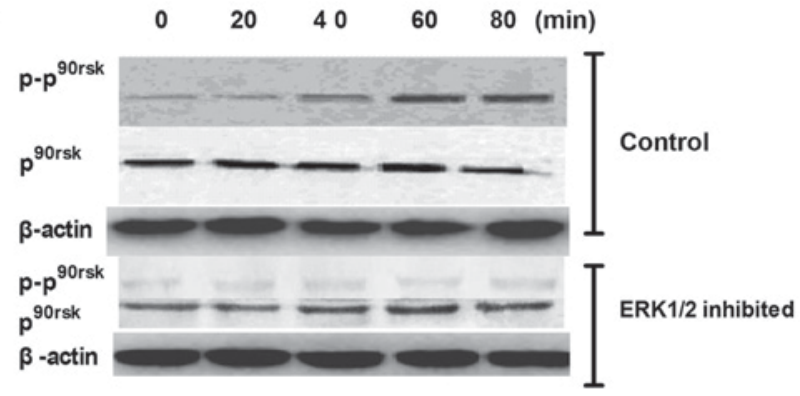

C

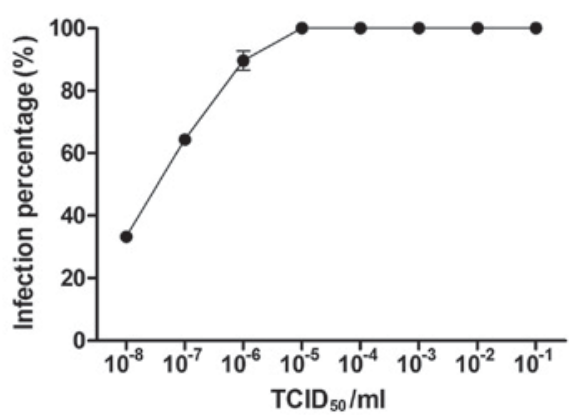

Figure 5. Effect of CVB3 infection on ERK1/2 and Rsk90 expression after ERK1/2 inhibition. (A) Representative western blots showing levels of total and phosphorylated ERK1/2. (B) Representative western blots showing levels of total and phosphorylated Rsk90. (C) TCID $_{50}$ of CVB3 in myocardial microvascular endothelial cells. TCID ${ }_{50}, 50 \%$ tissue culture infective dose CVB3, Coxsackieviruses B3; ERK, extracellular signal-regulated kinase.

\section{Discussion}

Viral myocarditis has been recognized as a cause of heart failure and premature death, but diagnosis is challenging; therefore, epidemiological studies of this condition are limited (9). During the course of infection by CVB3, a causative agent of viral myocarditis, primary replication occurs in the cells of the nasopharynx and the Peyer's patches of the intestine. Next, viruses spread via the blood circulation and interact inevitably with the vascular endothelium to reach target organs like the myocardium (8). Only a few publications have assessed infection of human endothelial cells by CVB3 $(21,22)$. Viruses interact with cell surface proteins that play integral roles in the biology of the target cell. Endothelial cells are exquisitely capable of responding to changes in their environment such as tissue damage and infection $(23,24)$.

Few studies of coxsackieviral infection of the endothelium have been published. Recently, it was shown that CVs infect polarized brain microvasculature and aortic endothelial cells (8); CV infection was associated with altered calcium signaling, which may be involved in mechanisms enabling the virus to cross endothelial barriers. Moreover, RNAi screening methods have shown that $\mathrm{CV}$ and poliovirus infections induce numerous alterations in brain endothelial

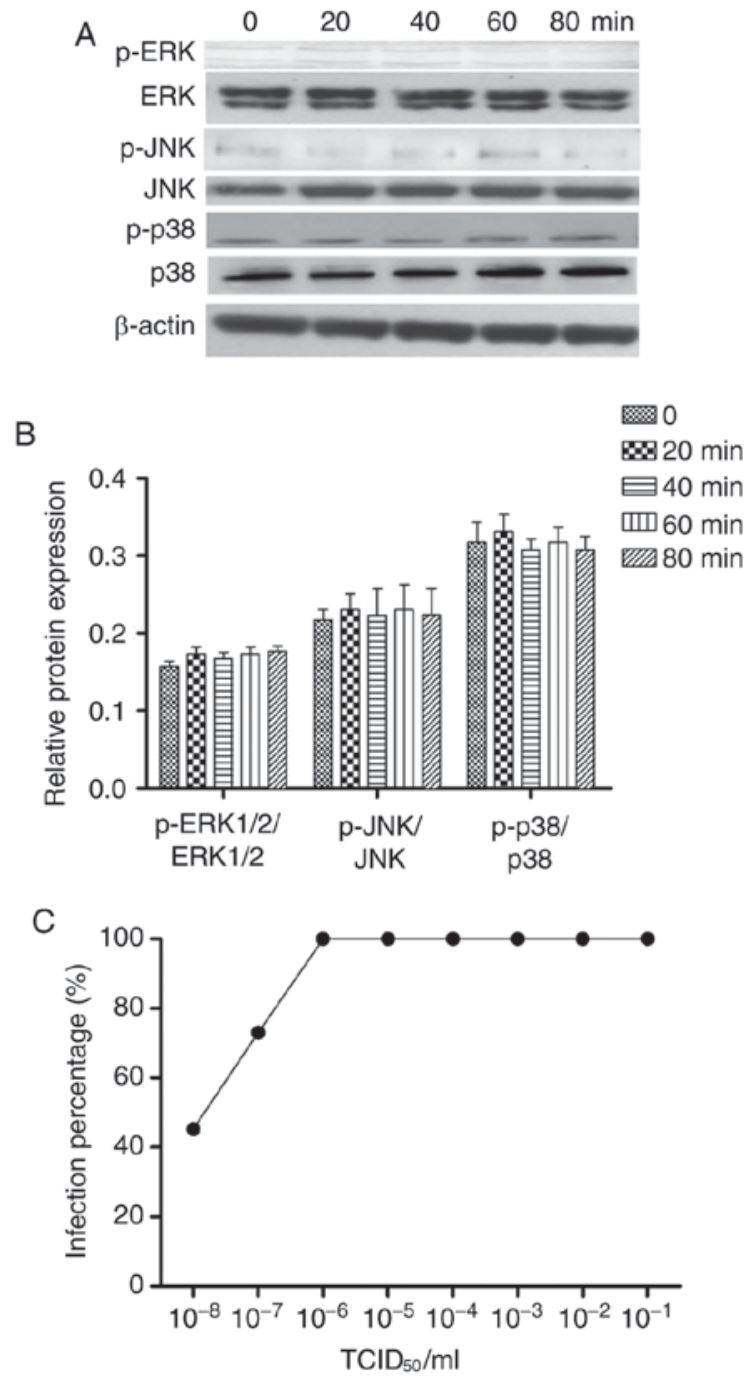

Figure 6. Effect of CVB3 infection on MAPK activation after FKN silencing. (A) Representative western blots showing levels of total and phosphorylated ERK, JNK, and p38. (B) Histograms summarizing the results shown in (A). Results are expressed as mean \pm standard deviation $(n=5)$. (C) TCID $_{50}$ of CVB3 in myocardial microvascular endothelial cells. TCID ${ }_{50}, 50 \%$ tissue culture infective dose; CVB3, Coxsackieviruses B3; FKN, fractalkine; ERK, extracellular signal-regulated kinase; JNK, c-Jun N-terminal kinase; MAPK, mitogen-activated protein kinase.

cells (25). CVB3 replication in rat cardiac microvascular endothelial cells alters expression of numerous cellular factors in a manner that may contribute to the progression of cardiac fibrosis (2).

In this study, CVB3 increased the expression of phosphorylated ERK1/2 in infected myocardial microvascular endothelial cells, while expression of FKN, a CX3C chemokine first identified in endothelial cells, was increased. FKN acts as a chemoattractant and adhesion molecule (10). Subsequent research identified several additional cellular sources of FKN, including neurons, microglial cells, osteoblasts, smooth muscle cells, dendritic cells, lymphocytes, and macrophages (11). The membrane-bound form of FKN functions as an adhesion protein, whereas soluble FKN acts as a chemokine (26). MAPKs ERK1/2, p38, and JNK play significant roles in regulating the synthesis of inflammatory mediators such as $\mathrm{NF}-\kappa \mathrm{B}$ and activator protein-1. 
Conversely, the production of inflammatory mediators is completely blocked by the suppression of multiple MAPK family members $(16,27)$. In this study, the total ERK1/2 protein level was increased.

To better understand the role of MAPK signaling in CVB3 infection, ERK1/2 was inhibited with U1206. The TCID $_{50}$ of CVB3 in ERK1/2-inhibited cells was higher than that of ERK1/2 uninhibited cells. In addition, there was no change in the expression of phosphorylated Rsk90, while FKN expression was inhibited.

CVB3 infection of myocardial microvascular endothelial cells activates FKN expression (against infetious) via the ERK1/2 signaling pathway. These findings may lead to the development of new treatments for patients with CVB3-induced myocarditis.

\section{References}

1. Zhou X, Xin Q, Wang Y, Zhao Y, Chai H, Huang X, Tao X and Zhao M: Total flavonoids of astragalus plays a cardioprotective role in viral myocarditis. Acta Cardiol Sin 32: 81-88, 2016.

2. Yu Y, Yu Y, Liu M, Yu P, Liu G, Liu Y, Su Y, Jiang H and Chen R Ethyl pyruvate attenuated coxsackievirus B3-induced acute viral myocarditis by suppression of HMGB1/RAGE/NF-KB pathway. Springerplus 5: 215, 2016.

3. Rose NR: Viral myocarditis. Curr Opin Rheumatol 28: 383-389, 2016.

4. Sun XH, Fu J and Sun DQ: Halofuginone alleviates acute viral myocarditis in suckling BALB/c mice by inhibiting TGF- $\beta 1$. Biochem Biophys Res Commun 473: 558-564, 2016.

5. Huber SA: Viral myocarditis and dilated cardiomyopathy: Etiology and pathogenesis. Curr Pharm Des 22: 408-426, 2016.

6. Werner L, Deutsch V, Barshack I, Miller H, Keren G and George J: Transfer of endothelial progenitor cells improves myocardial performance in rats with dilated cardiomyopathy induced following experimental myocarditis. J Mol Cell Cardiol 39: 691-697, 2005.

7. Wen C, Xie G, Zeng P, Huang LF and Chen CY: Tranilast inhibits myocardial fibrosis in mice with viral myocarditis. Zhongguo Dang Dai Er Ke Za Zhi 18: 446-454, 2016 (In Chinese).

8. Li-Sha G, Jing-Lin Z, Guang-Yi C, Li L, De-Pu Z and Yue-Chun L: Erratum: Dose-dependent protective effect of nicotine in a murine model of viral myocarditis induced by coxsackievirus B3. Sci Rep 5: 17247, 2015.

9. Zhu H, Lou C and Liu P: Interleukin-27 ameliorates coxsackievirus-B3-induced viral myocarditis by inhibiting Th17 cells. Virol J 12: 189, 2015.

10. Xu MX, Yu R, Shao LF, Zhang YX, Ge CX, Liu XM, Wu WY, Li JM and Kong LD: Up-regulated fractalkine (FKN) and its receptor CX3CR1 are involved in fructose-induced neuroinflammation: Suppression by curcumin. Brain Behav Immun 58: 69-81, 2016.

11. Poniatowski ŁA, Wojdasiewicz P, Krawczyk M, Szukiewicz D, Gasik R, Kubaszewski Ł and Kurkowska-Jastrzębska I: Analysis of the role of CX3CL1 (Fractalkine) and its receptor CX3CR1 in traumatic brain and spinal cord injury: Insight into recent advances in actions of neurochemokine agents. Mol Neurobiol 54 2167-2188, 2017.

12. Julia V, Staumont-Salle D and Dombrowicz D: Role of fractalkine/CX3CL1 and its receptor CX3CR1 in allergic diseases. Med Sci (Paris) 32: 260-266, 2016.
13. Bagci B, Bagci G, Huzmeli C, Sezgin I and Ozdemir O: Associations of fractalkine receptor (CX3CR1) and CCR5 gene variants with hypertension, diabetes and atherosclerosis in chronic renal failure patients undergoing hemodialysis. Int Urol Nephrol 48: 1163-1170, 2016.

14. Zanier ER, Marchesi F, Ortolano F, Perego C, Arabian M, Zoerle T, Sammali E, Pischiutta F and De Simoni MG: Fractalkine receptor deficiency is associated with early protection but late worsening of outcome following brain trauma in mice. J Neurotrauma 33: 1060-1072, 2016.

15. Kim SM, Kim H, Jang KW, Kim MH, Sohn J, Yun MR, Kang HN, Kang CW, Kim HR, Lim SM, et al: EGFR-mediated reactivation of MAPK signaling induces acquired resistance to GSK2118436 in BRAF V600E mutant NSCLC cell lines. Mol Cancer Ther 15: 1627-1636, 2016.

16. Chandaka GK, Wang L, Senogles S and Armstrong WE: Late pregnancy is a critical period for changes in phosphorylated mitogen-activated protein kinase/extracellular signal-regulated kinase 1/2 in oxytocin neurons. J Neuroendocrinol: doi: 10.1111/ jne. 12398

17. Itoh S, Ding B, Bains CP, Wang N, Takeishi Y, Jalili T, King GL, Walsh RA, Yan C and Abe J: Role of p90 ribosomal S6 kinase (p90RSK) in reactive oxygen species and protein kinase $\mathrm{C}$ beta (PKC-beta)-mediated cardiac troponin I phosphorylation. J Biol Chem 280: 24135-24142, 2005.

18. Roux PP, Richards SA and Blenis J: Phosphorylation of p90 ribosomal S6 kinase (RSK) regulates extracellular signal-regulated kinase docking and RSK activity. Mol Cell Biol 23: 4796-4804, 2003.

19. Neise D, Sohn D, Stefanski A, Goto H, Inagaki M, Wesselborg S, Budach W, Stühler K and Jänicke RU: The p90 ribosomal S6 kinase (RSK) inhibitor BI-D1870 prevents gamma irradiation-induced apoptosis and mediates senescence via RSK- and p53-independent accumulation of p21WAF1/CIP1. Cell Death Dis 4: e859, 2013.

20. Dou Zhongxia ZX: Role of NF-kappa B pathway in viral myocarditis in mice. J Nanchang Univ (Med Sci), 2016.

21. Ju Y, Wang T, Li Y, Xin W, Wang S and Li J: Coxsackievirus B3 affects endothelial tight junctions: possible relationship to ZO-1 and F-actin, as well as p38 MAPK activity. Cell Biol Int 31: 1207-1213, 2007.

22. Dai Q, Zhang D, Yu H, Xie W, Xin R, Wang L, Xu X, He X, Xiong J, Sheng H, et al: Berberine restricts coxsackievirus B type 3 replication via inhibition of c-Jun N-Terminal Kinase (JNK) and p38 MAPK Activation in vitro. Med Sci Monit 23: 1448-1455, 2017.

23. Liu J, Zhao J, Petrochenko P, Zheng J and Hewlett I: Sensitive detection of influenza viruses with Europium nanoparticles on an epoxy silica sol-gel functionalized polycarbonate-polydimethylsiloxane hybrid microchip. Biosens Bioelectron 86: 150-155, 2016.

24. Cusinato R, Pacenti M, Martello T, Fattori P, Morroni M and Palù G: Effectiveness of hydrogen peroxide and electron-beam irradiation treatment for removal and inactivation of viruses in equine-derived xenografts. J Virol Methods 232: 39-46, 2016.

25. Elmastour F, Jaidane H, Aguech-Oueslati L, Benkahla MA, Aouni M, Gharbi J, Sane F and Hober D: Immunoglobulin G-dependent enhancement of the infection with Coxsackievirus B4 in a murine system. Virulence 7: 527-535, 2016.

26. Merino JJ, Muñetón-Gómez V, Alvárez MI and Toledano-Díaz A: Effects of CX3CR1 and fractalkine chemokines in amyloid beta clearance and p-Tau Accumulation in Alzheimer's disease (AD) rodent models: Is fractalkine a systemic biomarker for AD? Curr Alzheimer Res 13: 403-412, 2016.

27. Rodriguez-Carballo E, Gámez B and Ventura F: p38 MAPK signaling in osteoblast differentiation. Front Cell Dev Biol 4: 40, 2016. 